\title{
Minimum-Latency Beaconing Schedule in Duty-Cycled Multihop Wireless Networks
}

\author{
Lixin Wang*, Peng-Jun Wan ${ }^{\dagger}$, and Kyle Young ${ }^{\ddagger}$ \\ * Department of Mathematics, Sciences and Technology, Paine College, Augusta, GA 30901, lwang@paine.edu \\ $\dagger$ Department of Computer Science, Illinois Institute of Technology, Chicago, IL 60616, wan@cs.iit.edu \\ $\ddagger$ Department of Mathematics, Sciences and Technology, Paine College, Augusta, GA 30901, youngko@ paine.edu
}

\begin{abstract}
Beaconing is a primitive communication task in which every node locally broadcasts a packet to all of its neighbors within a fixed distance. The problem Minimum Latency Beaconing Schedule (MLBS) seeks a shortest schedule for beaconing subject to the interference constraint. MLBS has been well studied when all the nodes are always awake. However, it is wellknown that the networking nodes often switch between the active state and the sleep state to save energy. A node in duty-cycled scenarios may require transmitting multiple times to inform all of its neighbors due to their different active times. Thus, all of the known algorithms for MLBS are not suitable for duty-cycled multihop wireless networks. In this paper, we study MLBS in Duty-Cycled multihop wireless networks (MLBSDC). We first present two constant-approximation algorithms for MLBSDC under the protocol interference model with the approximation bounds independent of the length of a scheduling period. Then, we develop an efficient algorithm for MLBSDC under the physical interference model. To the best of our knowledge, this is the first paper that develops efficient algorithms for MLBSDC under either of these two interference models.
\end{abstract}

Keywords-Beaconing schedule, duty cycle, approximation algorithms, protocol interference model, physical interference model.

\section{INTRODUCTION}

Beaconing in multihop wireless networks is a primitive communication task in which every node locally broadcasts a packet to all of its neighbors. In order for all the networking nodes to collect information from their neighboring nodes, beaconing is often used in many network protocols. Therefore, seeking a fast beaconing schedule is not only of theoretical interest, but also of practical importance. Assume that all communications proceed in synchronous time-slots and each node can transmit at most one packet of a fixed size in each time-slot. A beaconing schedule assigns a timeslot to every node subject to the constraint that the nodes assigned in each time-slot are interference free. The problem of computing a beaconing schedule with minimum latency is referred to Minimum-Latency Beaconing Schedule (MLBS). MLBS is NP-hard even in the simplest setting: all the nodes have uniform beaconing radius and uniform interference radius equal to the beaconing radius [12]. MLBS and its variants have been extensively studied in the literature [1], [2], [4], [10], [11], [12], [13], [14], [15], [17], [18].

However, it is well-known that the nodes often switch between the active state and the sleep state to save energy. The duty-cycled scenarios have been emerging as a prevalent energy-saving method in multihop wireless networks. A node in duty-cycled scenarios with active/sleep cycles may require transmitting multiple times to inform all of its neighbors due to their different active times. Thus, all of the previous known beaconing scheduling algorithms are not suitable when dutycycled scenarios are taken into consideration.

In this paper, we study the MLBS problem in DutyCycled multihop wireless networks (MLBSDC) under both the protocol interference model and the physical interference model. Under the protocol interference model, assume that every node $v$ has a unit beaconing radius and its beaconing range is the unit disk centered at $v$. In addition, each node $v$ has an interference radius $\rho(v) \geq 1$ and its interference range is the disk centered at $v$ of radius $\rho(v)$. Then, a pair of nodes $u$ and $v$ conflict with each other (i.e., they cannot transmit simultaneously) if and only if one of the following three conditions holds (see [15]): (1) $u$ and $v$ are within each other's beaconing range, (2) some node $w$ other than $u$ and $v$ is within $u$ 's beaconing range and $v$ 's interference range, and (3) some node $w$ other than $u$ and $v$ is within $v$ 's beaconing range and $u$ 's interference range.

Under the physical interference model, assume all the nodes transmit at a fixed power $P$. The path loss is determined by a positive reference loss parameter $\eta$, and the path-loss exponent $\kappa$. Specifically, when a node $u$ transmits at power $P$, the power of this signal captured by another node $v$ is $\eta P\|u v\|^{-\kappa}$, where $\|u v\|$ denotes the scaled Euclidean distance between $u$ and $v$ so that the the beaconing radius is normalized to one. The signal quality perceived by a receiver is measured by the signal to interference and noise ratio (SINR), which is the ratio between the power of the wanted signal and the total power of unwanted signals plus the background noise $\xi$. In 
order to correctly interpret the wanted signal, the SINR must be no less than certain threshold $\sigma$. The physical interference model is also referred to as the SINR model.

With the duty-cycled scenarios, assume that all the nodes determine the active/sleep time without coordination in advance, and thus do not require additional communication overhead. The duty cycle is defined as the ratio of the active time to the whole scheduling time. The whole scheduling time is divided into multiple scheduling periods of the same length. A scheduling period $T$ is further divided into fixed $|T|$ time slots, i.e., $T=\{0,1, \cdots,|T|-1\}$. Every node $v$ chooses exactly one active time slot in $T$ randomly and independently, and wakes up at this time slot in every scheduling period to receive the message. If a node $v$ needs to send a message as required, it can wake up at any time slot to transmit as long as the receiving node is awake and there is no collision for this transmission.

The contributions of this paper are listed below:

(1) This is the first paper that develops efficient algorithms for the problem MLBSDC under either the protocol interference model or the physical interference model;

(2) Under the protocol interference model, we first propose an efficient first-fit 74-approx. algorithm for MLBSDC when all nodes have arbitrary interference radii. This algorithm achieves approximation bound at most 29 when all nodes have uniform interference radius $\rho>1$, and at most 15 when $\rho=1$. Then we develop another efficient strip-coloring constant-approx. algorithm for MLBSDC with all nodes having uniform interference radius $\rho \geq 1$. This algorithm achieves approximation bound at most 10 when $\rho=1$, and between 6 and 12 in general;

(3) With the duty-cycled scenarios, the approximation bounds of all the known scheduling algorithms for any communication tasks (e.g., broadcast, data aggregation, etc.) depend on $|T|$, the length of a scheduling period (see the related works in Section II for details). This is the first paper that develops constant-approx. algorithms for communication scheduling with the approximation bound independent of $|T|$ when the duty-cycled scenarios are taken into consideration;

(4) Under the physical interference model, we develop an efficient first-fit approximation algorithm for MLBSDC.

All the three algorithms developed in this paper for MLBSDC have efficient implementations and can be used as a part of the procedures in many network protocols that requires the nodes collecting information from their neighboring nodes in any time-critical multihop wireless networks with duty-cycled scenarios.
The remaining of this paper is organized as follows. In Section II, we give a literature review for the related works. In Section III, we present two efficient constant-approx. algorithms for MLBSDC under the protocol interference model. Another efficient approximation algorithm for MLBSDC under the physical interference model is proposed in Section IV. Finally, we conclude our paper and discuss some future research directions in Section V.

\section{RELATED WORKS}

MLBS and its variants have been well studied in the literature. Most prior works [1][2][4][10][11][12][13][14] adopted the protocol interference model and assumed that all the nodes have uniform beaconing radius and uniform interference radius equal to the beaconing radius. Based on the first-fit coloring algorithm proposed in [9], Sen et al. [13] presented an algorithm for MLBS by computing a vertex coloring of the square of the unit-disk graph. This algorithm has approximation bound at most 7 that was proved by Wan et al. in [18]. When all the nodes have uniform interference radius $\rho \geq 1$, Wan et al. [17] developed a strip-coloring algorithm for MLBS that has approximation ratio between 3 and 6 in general, and at most 5 when $\rho=1$. When each node $v$ has arbitrary interference radius $\rho(v) \geq 1$, Wan et al. [15] proposed an first-fit 61-approx. algorithm for MLBS that achieves approximation bound at most 25 when all nodes have uniform radius $\rho \geq 1$, and at most 7 when $\rho=1$. The above known algorithms on MLBS are all obtained under the protocol interference model. Under the SINR model, Wan et al. [15] proposed a first-fit constantapprox. algorithm for MLBS. All these known algorithms on MLBS are obtained under the assumption that the networking nodes are always active.

With the duty-cycled scenarios, Han et al. [7] provided a survey on various algorithms designed for data communications in multihop wireless networks and discussed different duty-cycling models used by existing works. Hong et al. [6], Jiao et al. [5], Xu et al. [19] proposed approximation algorithms for the one-to-all broadcast scheduling with the duty-cycled scenarios. Their algorithms respectively achieve approximation ratios at most $24|T|+1,17|T|$, and $(1+$ $o(1))|T|$. Jiao et al. [5] also proposed two algorithms for the all-to-all broadcast scheduling with approximation ratios at most respectively $17|T|+20$ and $(\Delta+22)|T|$, where $\Delta$ is the maximum node degree. Xu et al. [19] proposed an algorithm for data aggregation of total latency at most $(6 \Delta+3 R+O(\log R))|T|$, where $R$ is the radius of the communication network w.r.t. the sink node, and an algorithm for gossiping schedule of approximation bound at most $20|T|$, and a scheduling algorithm for data collection with approximation bound at most $10|T|$. All these existing algorithms 
with the duty-cycled scenarios described above were developed under the protocol interference model.

To the best of our knowledge, efficient approximation algorithms for the problem MLBSDC have not yet be explored under any interference model. This paper develops efficient approximation algorithms for MLBSDC under both the protocol interference model and the SINR model.

\section{BEACONING SCHEDULE WITH DUTY-CYCLED SCENARIOS SUbJECT TO PROTOCOL INTERFERENCE}

Consider an instance of a multihop wireless network with duty-cycled scenarios under the protocol interference model specified by a finite planar set $V$ of nodes together with unit beaconing radius and an arbitrary interference radius $\rho(v) \geq 1$ for each node $v \in V$. We define the conflict graph $H$ over the vertices of $V$ as follows: For any pair of nodes $u$ and $v$, there is an edge between $u$ and $v$ in the conflict graph $H$ if and only if they cannot transmit simultaneously (i.e., they interference with each other). Then, a beaconing schedule for $V$ under the protocol interference model is equivalent to a vertex coloring of the conflict graph $H$ with the latency corresponding to the number of colors.

In this section, we develop constant-approx. algorithms to computer a beaconing schedule for MLBSDC under the protocol interference model with either arbitrary interference radii or uniform interference radii. For any $u \in V$, let $N(u) \subseteq V \backslash\{u\}$ denote the set of all neighbors of $u$. Recall that $|T|$ denotes the total number of time slots in any scheduling period $T$. For each time slot $0 \leq i \leq|T|-1$, let $U_{i}$ denote the set of all nodes in $V$ that are active in the time slot $i$ and

$$
N\left(U_{i}\right)=\bigcup_{u \in U_{i}} N(u)
$$

Next we analyze a lower bound on the latency of any optimal beaconing schedule for the problem MLBSDC under the protocol interference model. Clearly, a trivial lower bound on the latency of any optimal beaconing schedule for such a problem is $\chi(H)$, the chromatic number of $H$. The following lemma gives a better lower bound in terms of $|T|$ and the clique number $\omega(H)$ of $H$.

Lemma 1: A lower bound on the latency of any optimal beaconing schedule for the problem MLBSDC under the protocol interference model is at least $(\omega(H)-1)|T|$.

Proof: Let $C$ denote a maximum clique of the conflict graph $H$. Then $|C|=\omega(H)$. Let $S_{o p t}$ denote any optimal beaconing schedule for the problem MLBSDC under the protocol interference model. We consider two cases:

Case 1. All the nodes in $C$ have pairwise distinct active time-slots. Then no two nodes in $C$ share the same active timeslot. Without loss of generality, assume the $\omega(H)$ nodes in $C$ are respectively active at the time-slots $0,1,2, \cdots, \omega(H)-1$. Then at each time-slot $0 \leq i \leq \omega(H)-1$, exactly one node in $C$ is active and ready to receive a message. Thus, at each time-slot $0 \leq i \leq \omega(H)-1$, exactly $\omega(H)-1$ nodes in $C$ must be scheduled for transmission. Since $C$ is a clique of $H$, no two nodes in $C$ can transmit simultaneously. Therefore, in any optimal beaconing schedule $S_{o p t}$, for each time-slot $0 \leq i \leq \omega(H)-1$, the number of times that the time-slot $i$ is used for reception is at least $\omega(H)-1$. Hence, a lower bound on the latency of the optimal beaconing schedule $S_{\text {opt }}$ is at least $(\omega(H)-1)|T|$ in this case.

Case 2. At least two nodes in $C$, say $u$ and $v$, share the same active time-slot $i_{0}$ for some $0 \leq i_{0} \leq|T|-1$. Then every node in $C$, including $u$ and $v$, has at least one active neighbor in $C$ at the time-slot $i_{0}$. Thus, every node in $C$ must be scheduled to transmit at least once at the time-slot $i_{0}$. Since $C$ is a clique $H$, no two nodes in $C$ can transmit simultaneously. Therefore, in any optimal beaconing schedule $S_{\text {opt }}$, the number of times that the time-slot $i_{0}$ is used for reception is at least $\omega(H)$. Hence, a lower bound on the latency of the optimal beaconing schedule $S_{\text {opt }}$ is at least $(\omega(H)-1)|T|+i_{0}$ in this case. Note that $i_{0} \geq 0$. Thus, the lemma holds in this case.

This completes the proof of the lemma.

\section{A. First-Fit Beaconing Schedule with Arbitrary Interference Radii}

In this subsection, we assume that each node $v \in V$ has an arbitrary interference radius $\rho(v) \geq 1$. We adopt the first-fit coloring which is described below: Given a vertex ordering $\left\langle v_{1}, v_{2}, \cdots, v_{n}\right\rangle$ of $V$, a coloring of $V$ with colors represented by natural numbers can be produced in the following firstfit manner: Assign the color 1 to $v_{1}$. For $i=2$ up to $n$, assign to $v_{i}$ with the smallest color which is not used by any preceding neighbor of $v_{i}$ in the ordering. Such coloring of $V$ is referred to as the first-fit coloring in the ordering $\left\langle v_{1}, v_{2}, \cdots, v_{n}\right\rangle$. It is easy to verify that the number of colors used by the first-fit coloring in the ordering $\left\langle v_{1}, v_{2}, \cdots, v_{n}\right\rangle$ is at most $1+\max _{1<i \leq n}\left|N_{<}\left(v_{i}\right)\right|$, where each $N_{<}\left(v_{i}\right)$ with $1<i \leq n$ consists of all preceding neighbors of $v_{i}$ in the ordering (i.e., all neighbors $v_{j}$ of $v_{i}$ with $1 \leq j<i$ ). The value $\max _{1<i \leq n}\left|N_{<}\left(v_{i}\right)\right|$, denoted by $\delta^{*}$, is referred to as the inductivity of the ordering $\left\langle v_{1}, v_{2}, \cdots, v_{n}\right\rangle$.

When all the networking nodes are always active and each node $v \in V$ has an arbitrary interference radius $\rho(v) \geq 1$, 
Wan et al. [15] utilized the First-Fit coloring and developed a Beaconing Scheduling subject to Protocol interference (FFBSPr). The beaconing schedule produced by this first-fit algorithm uses at most $61 \chi(H)-60$ colors, where $\chi(H)$ is the chromatic number of the conflict graph $H$.

By Lemma 1, a lower bound on the latency of any optimal beaconing schedule for the problem MLBSDC under the protocol interference model is represented in terms of $\omega(H)$ and $|T|$. Next we derive a better upper bound for the first-fit algorithm FFBS-Pr proposed in [15] by providing an upper bound for the inductivity $\delta^{*}(H)$ of the conflict graph $H$ in the interference radius decreasing ordering of $V$.

Theorem 2: The first-fit beaconing schedule produced by the algorithm FFBS-Pr in [15] under the protocol interference model uses at most $\min \{61 \chi(H)-60,73 \omega(H)-72\}$ colors.

Note that for any graph $G$, we have $\chi(G) \geq \omega(G)$ with the equality holds when $G$ is perfect. But the ratio $\chi(G) / \omega(G)$ could be arbitrarily large for some graphs. Therefore, when the ratio $\chi(G) / \omega(G)$ is large, Theorem 2 gives a much better upper bound for the total latency of the first-fit beaconing schedule produced by the algorithm FFBS-Pr proposed in [15] under the protocol interference model. In order to prove this theorem, we need the following geometric lemma:

Lemma 3: Given a disk $D$ of radius 3 centered at any point $o$. Then the disk $D$ can be partitioned into at most 54 pieces, each of which has diameter at most one.

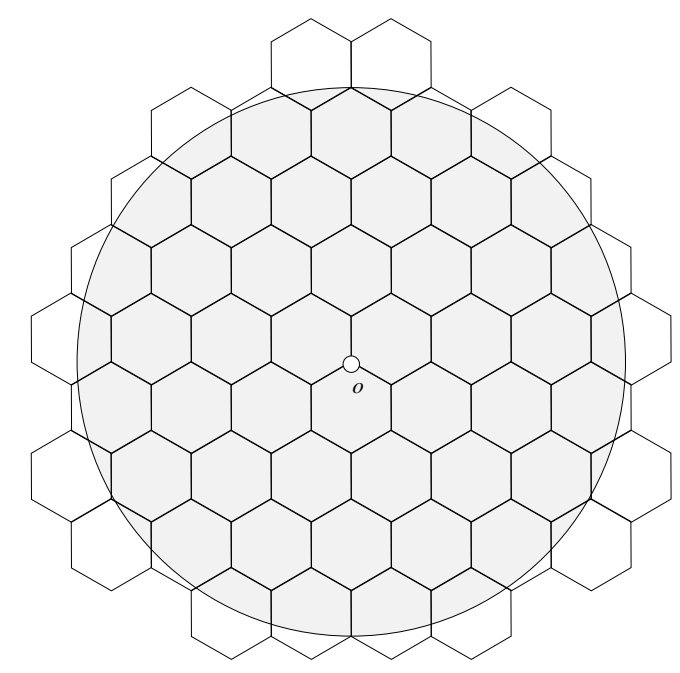

Fig. 1. 54 hexagons fully or partially contained in $D$ of radius 3 centered at $o$.

Proof: We tile the plane into regular hexagons of diameter one such that the center $o$ is the common vertex of three adjacent hexagons (see Fig. 1). Note that each regular hexagon has side equal to $\frac{1}{2}$. Through a direct counting from the coverage for the disk $D$ of radius 3 centered at $o$ by unitdiameter regular hexagons, there are totally 54 hexagons fully or partially contained in $D$. Thus, the lemma holds.

The proof of Theorem 2 is long and it is omitted in this conference version of the paper due to the page limitation. Interested readers can refer to the full version of this paper [16] available online.

Now we are ready to describe the algorithm for the FirstFit Beaconing Scheduling with Duty-cycled scenarios under the Protocol interference model (FFBSD-Pr). At time slot 0, we apply the first-fit algorithm FFBS-Pr proposed in [15] on the induced subgraph $H\left[N\left(U_{0}\right)\right]$ of $H$. The nodes in $N\left(U_{0}\right)$ is then partitioned into $k_{0}$ independent sets $I_{01}, I_{02}, \cdots, I_{0 k_{0}}$ for some integer $k_{0}>0$. For each $1 \leq j \leq k_{0}$, the nodes in $I_{0 j}$ transmit at the time slot 0 in the $j$-th scheduling period. In general, at each time slot $i(0 \leq i \leq|T|-1)$, we apply the firstfit algorithm FFBS-Pr on the induced subgraph $H\left[N\left(U_{i}\right)\right]$ of $H$, the nodes in $N\left(U_{i}\right)$ is then partitioned into $k_{i}$ independent sets $I_{i 1}, I_{i 2}, \cdots, I_{i k_{i}}$ for some integer $k_{i}>0$. For each $1 \leq$ $j \leq k_{i}$, the nodes in $I_{i j}$ transmit at the time slot $i$ in the $j$-th scheduling period.

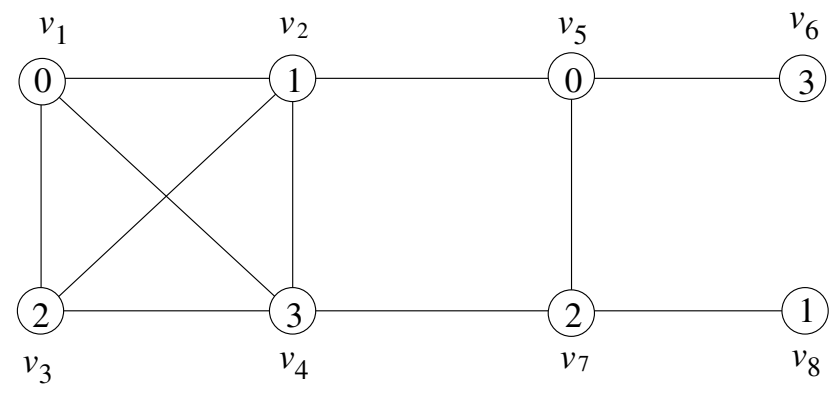

Fig. 2. An example to illustrate the algorithm FFBSD-Pr. The number inside a node represents the active time-slot of the node.

Example 1. We take the graph in Fig. 2 as an example to illustrate the first-fit scheduling algorithm FFBSD-Pr. The network consists of eight nodes as shown in the figure. In this example, we assume that all eight nodes have uniform beaconing radius normalized to one and uniform interference radius equal to $\rho=1$ for simplicity. Then the interference graph $H$ is exactly the square of the communication graph shown in Fig. 2. That is, two nodes in $H$ are directly connected if and only if they are at most two-hop away in the communication topology.

The scheduling period $T=\{0,1,2,3\}$. The number inside a node represents the active time slot of the node. At time slot $0, U_{0}=\left\{v_{1}, v_{5}\right\}$ and $N\left(U_{0}\right)=\left\{v_{2}, v_{3}, v_{4}, v_{6}, v_{7}\right\}$. We apply the first-fit algorithm FFBS-Pr proposed in [15] on the induced subgraph $H\left[N\left(U_{0}\right)\right]$ of $H$. The nodes in $N\left(U_{0}\right)$ is then partitioned into three independent sets $I_{01}=\left\{v_{2}\right\}, I_{02}=$ 


\begin{tabular}{|l|l|l|l|l|}
\hline & time-slot 0 & time-slot 1 & time-slot 2 & time-slot 3 \\
\hline$S P 1$ & $\left\{v_{2}\right\}$ & $\left\{v_{1}, v_{7}\right\}$ & $\left\{v_{1}, v_{5}\right\}$ & $\left\{v_{1}, v_{5}\right\}$ \\
\hline$S P 2$ & $\left\{v_{3}, v_{6}\right\}$ & $\left\{v_{3}\right\}$ & $\left\{v_{2}, v_{8}\right\}$ & $\left\{v_{2}\right\}$ \\
\hline$S P 3$ & $\left\{v_{4}, v_{7}\right\}$ & $\left\{v_{4}\right\}$ & $\left\{v_{4}\right\}$ & $\left\{v_{3}\right\}$ \\
\hline$S P 4$ & & $\left\{v_{5}\right\}$ & & $\left\{v_{7}\right\}$ \\
\hline
\end{tabular}
ALGORITHM FFBSD-PR FOR THE GRAPH IN EXAMPLE 1. IN EACH ROW, "SP I" STANDS FOR THE I-TH SCHEDULING PERIOD.

$\left\{v_{3}, v_{6}\right\}$, and $I_{03}=\left\{v_{4}, v_{7}\right\}$. The nodes in $I_{01}, I_{02}$, and $I_{03}$ are scheduled to transmit at the time-slot 0 in the Scheduling Periods (SP) 1,2 and 3, respectively. Similarly, at each time slot $i(1 \leq i \leq 3)$, we apply the first-fit algorithm FFBSPr on the induced subgraph $H\left[N\left(U_{i}\right)\right]$ of $H$. The beaconing schedule produced by the algorithm FFBSD-Pr is listed in the following Table I. Each row lists the sets of nodes will be scheduled to transmit at the specified Scheduling Period (SP). Each column lists the sets of nodes that will be scheduled to transmit at the specified time-slot. The set of nodes in each entry is scheduled to transmit at the time-slot specified by the column at the scheduling period specified by the row. For example, the set of nodes $\left\{v_{3}, v_{6}\right\}$ is scheduled to transmit at time-slot 0 of the scheduling period 2 (SP 2). Totally, four scheduling periods are required for all the eight nodes to complete their beaconing operations based on the algorithm FFBSD-Pr.

The next theorem asserts the correctness of the above algorithm FFBSD-Pr and establishes a constant approximation bound for this algorithm.

Theorem 4: The first-fit beaconing schedule produced by the above algorithm FFBSD-Pr is correct and uses at most $\min \{61 \chi(H)-60,73 \omega(H)-72\} \cdot|T|$ colors. This algorithm achieves approximation bound at most 74 .

Proof: Let $K=\min \{61 \chi(H)-60,73 \omega(H)-72\}$. Every color is represented by a pair of positive integers $\left(\lambda_{1}, \lambda_{2}\right)$, where $\lambda_{1}$ represents a time slot in a scheduling period $T=\{0,1, \cdots,|T|-1\}$ and $\lambda_{2}$ represents one of the independent sets in the partition of the nodes in $N\left(U_{\lambda_{1}}\right)$ when applying the algorithm FFBS-Pr on the induced subgraph $H\left[N\left(U_{\lambda_{1}}\right)\right]$. Therefore, $0 \leq \lambda_{1} \leq|T|-1$ and $1 \leq \lambda_{2} \leq K$. Thus, the algorithm FFBSD-Pr uses at most $K|T|$ colors.

Next we prove the algorithm FFBSD-Pr is correct. Given any node $v \in V$. Assume that $v$ is active at the time slot $s$ for some $0 \leq s \leq|T|-1$. Then $v \in U_{s}$. When applying the algorithm FFBS-Pr on the induced subgraph $H\left[N\left(U_{s}\right)\right]$, the nodes in $N\left(U_{s}\right)$ is partitioned into independent sets $I_{s 1}, I_{s 2}, \cdots, I_{s k_{s}}$, where $k_{s} \leq K$. For each $1 \leq j \leq k_{s}$, the nodes in $I_{s j}$ transmit at the time slot $s$ in the $j$-th scheduling period. Since a sender can wake up at any time slot to transmit the message as long as the receiver node is awake and there is no collision for this transmission. Therefore, at the end of the $K$-th scheduling period, the node $v$ received the messages from all of its neighbors. Thus, at the end of the $K$-th scheduling period, the beaconing operations of all the networking nodes are complete.

By using the lower bound obtained in Lemma 1, the approximation bound for the above algorithm FFBSD-Pr is at most

$$
\begin{aligned}
& \frac{\min \{61 \chi(H)-60,73(\omega(H)-1)+1\} \cdot|T|}{(\omega(H)-1)|T|} \\
& \leq 73+1 /(\omega(H)-1) \leq 74 .
\end{aligned}
$$

This completes the proof for the theorem.

\section{B. Beaconing Schedule with Uniform Interference Radius}

In this subsection, we assume that all nodes in $V$ have uniform interference radius equal to $\rho \geq 1$. Under such an assumption, we first derive a better approximation bound for the first-fit algorithm FFBSD-Pr proposed above. Then we develop a new efficient algorithm to computer a beaconing schedule for MLBSDC under the protocol interference model that has better performance bound than FFBSD-Pr.

The first-fit beaconing schedule algorithm FFBS-Pr proposed in [15] has approximation bound at most $\mu\left(\frac{\rho+1}{\max \{1, \rho-1\}}\right)$ when $\rho>1$, and at most 7 when $\rho=1$ (see Theorem 9 in [15]), where $\mu(x)$ denote the maximum number of points in a half-disk of radius $x \geq 1$ whose mutual distances are greater than one. The total latency of the beaconing schedule produced by this algorithm is at most $\mu\left(\frac{\rho+1}{\max \{1, \rho-1\}}\right)(\chi(H)-1)+1$ when $\rho>1$, and at most $7(\chi(H)-1)+1$ when $\rho=1$ (see Theorem 2 in [15]).

Next we derive a better upper bound for the total latency of the beaconing schedule produced by the algorithm FFBS-Pr in the following theorem:

Theorem 5: Assume that all the nodes have uniform interference radius $\rho \geq 1$. Then the total latency of the beaconing schedule produced by FFBS-Pr proposed in [15] is at most $28 \omega(H)-27$ when $\rho>1$, and at most $14 \omega(H)-13$ when $\rho=1$.

In order to prove Theorem 5, we need the following geometric lemma which is similar to Lemma 3:

Lemma 6: (1) Any half-disk of radius 3 can be partitioned into at most 28 pieces, each of which has diameter at most one. (2) Any half-disk of radius 2 can be partitioned into at most 14 pieces, each of which has diameter at most one. 
The proofs of Lemma 6 and Theorem 5 are omitted in this conference version of the paper due to the page limitation. Interested readers can refer to the full version of this paper [16] available online.

By Theorem 5, we can obtain the improved performance bounds for our algorithm FFBSD-Pr proposed above when all the networking nodes have uniform interference radii $\rho \geq 1$ in the following theorem.

Theorem 7: Assume that all the nodes have uniform interference radius equal to $\rho \geq 1$. The performance bound of the algorithm FFBSD-Pr developed above is at most 15 when $\rho=1$, and at most 29 when $\rho>1$.

Proof: By Theorem 5, the total latency of the beaconing schedule produced by our algorithm FFBSD-Pr developed above is at most $(28 \omega(H)-27)|T|$ when $\rho>1$, and at most $(14 \omega(H)-13)|T|$ when $\rho=1$. Based on the lower bound obtained in Lemma 1, the performance ratio of this algorithm is at most 15 when $\rho=1$, and at most 29 when $\rho>1$. This completes the proof of the theorem.

Next we develop a new polynomial-time Strip-Coloring algorithm to computer a Beaconing Schedule with Duty-cycled scenarios subject to Protocol interference (SCBSD-Pr) for the problem MLBSDC that has better performance bound than FFBSD-Pr. We adopt the Strip-Coloring algorithm for Beaconing Scheduling under the Protocol interference model (SCBS-Pr) proposed in [17] as a part of the procedure in this new beaconing schedule algorithm.

Let $k(\rho)=\left\lceil\frac{\rho+1}{h(\rho)}\right\rceil$, where $h(\rho)$ is defined as follows:

$$
\begin{aligned}
& h(\rho)=\cos \frac{\arccos \frac{\rho}{2}+\arccos \frac{1}{2 \rho}}{2} \text { if } 1 \leq \rho<\sqrt{\frac{3+\sqrt{13}}{2}} ; \\
& h(\rho)=\sin \left(\arccos \frac{1}{2 \rho}-\arcsin \frac{1}{\rho}\right) \text { if } \sqrt{\frac{3+\sqrt{13}}{2}} \leq \rho \leq 2 ; \\
& h(\rho)=(\rho-1) \sin \left(\arccos \frac{\rho-1}{2 \rho}-\arcsin \frac{1}{\rho}\right) \text { if } \rho>2 .
\end{aligned}
$$

An upper and a lower bounds for $k(\rho)$ are given in the following lemma which was proved in Section II of [17].

Lemma 8: For any $\rho \geq 1$, we have $2 \leq k(\rho) \leq 5$. When $\rho=1, k(\rho)=4$.

In order to apply the algorithm SCBS-Pr proposed in [17], we first compute the minimal axis-parallel rectangle surrounding all the networking nodes. Then, we partition such rectangle into top-closed bottom-open horizontal strips in the manner that the upper boundary of the top-most strip aligns with the top of the rectangle, the heights of all strips except the bottom-most one are all equal to $\frac{\rho+1}{k(\rho)}$ (see Fig. 3). A key idea used in this algorithm is that if two nodes are separated by an Euclidean distance greater than $\rho+1$ ( 1 is the beaconing radius), then they do not interference with each other and are independent in the conflict graph $H$.

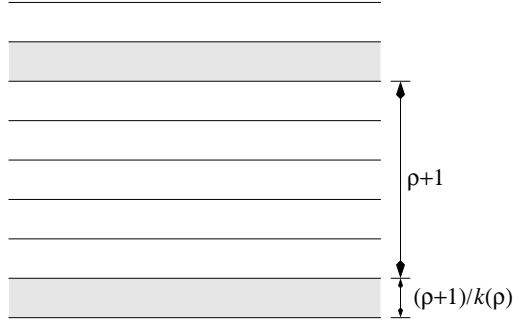

Fig. 3. Illustration of the strip-coloring algorithm.

The approximation bound of the strip-coloring algorithm SCBS-Pr, proved in [17], is given in the following lemma (see Theorem 7 in [17]):

Lemma 9: The strip-coloring algorithm SCBS-Pr proposed in [17] uses at most $(k(\rho)+1) \omega(H)$ colors, where $\omega(H)$ is the clique number of $H$. The algorithm has approximation bound at most $k(\rho)+1$.

Therefore, by Lemma 8 and Lemma 9, the algorithm SCBS-Pr achieves approximation bound at most 5 when $\rho=1$, and between 3 and 6 in general.

Now we are ready to describe the algorithm SCBSD-Pr to computer a beaconing schedule with duty-cycled scenarios. Let $Y=(k(\rho)+1) \omega(H)$. At time slot 0 , we apply the algorithm SCBS-Pr on the induced subgraph $H\left[N\left(U_{0}\right)\right]$. The nodes in $N\left(U_{0}\right)$ is then partitioned into $k_{0}$ independent sets $I_{01}, I_{02}, \cdots, I_{0 k_{0}}$, for some positive integer $k_{0} \leq Y$ by Lemma 9. For each $1 \leq j \leq k_{0}$, the nodes in $I_{0 j}$ transmit at the time slot 0 in the $j$-th scheduling period. In general, at each time slot $0 \leq i \leq|T|-1$, we apply the algorithm SCBSPr on the induced subgraph $H\left[N\left(U_{i}\right)\right]$, the nodes in $N\left(U_{i}\right)$ is then partitioned into $k_{i}$ independent sets $I_{i 1}, I_{i 2}, \cdots, I_{i k_{i}}$, for some positive integer $k_{i} \leq Y$. For each $1 \leq j \leq k_{i}$, the nodes in $I_{i j}$ transmit at the time slot $i$ in the $j$-th scheduling period.

Example 2. We take the graph in Fig. 4 as an example to illustrate the strip-wise coloring scheduling algorithm SCBSDPr. The network consists of twelve nodes as shown in the figure. In this example, we assume that all the twelve nodes have uniform beaconing radius normalized to one and uniform interference radius equal to $\rho=1$ for simplicity. It is easy to see that $h(\rho)=\frac{1}{2}$ and $k(\rho)=4$. The deployment region is partitioned into three horizontal strips and the height of each horizontal strip is $\frac{\rho+1}{k(\rho)}=\frac{1}{2}$ (see Fig. 4). Then the interference graph $H$ is exactly the square of the communication graph 


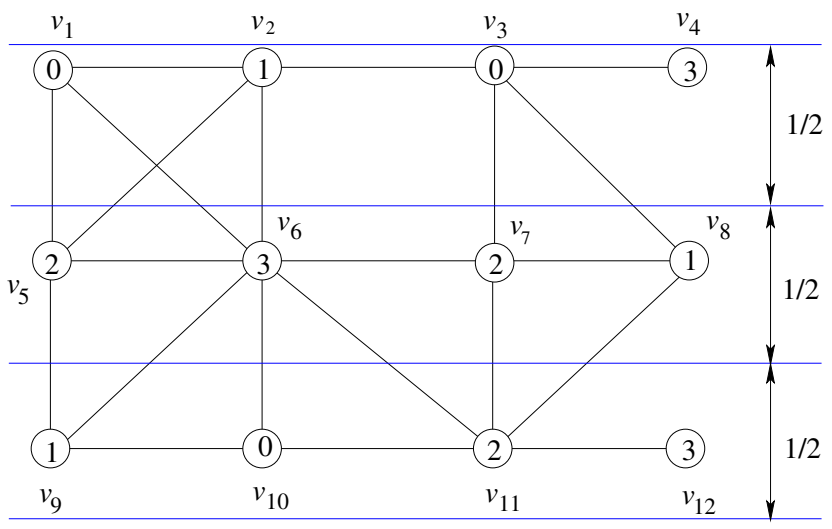

Fig. 4. An example to illustrate the algorithm SCBSD-Pr. The number inside a node represents the active time-slot of the node.

\begin{tabular}{|l|l|l|l|l|}
\hline & time-slot 0 & time-slot 1 & time-slot 2 & time-slot 3 \\
\hline$S P 1$ & $\left\{v_{2}\right\}$ & $\left\{v_{1}\right\}$ & $\left\{v_{1}, v_{3}\right\}$ & $\left\{v_{1}, v_{3}\right\}$ \\
\hline$S P 2$ & $\left\{v_{4}\right\}$ & $\left\{v_{3}\right\}$ & $\left\{v_{2}\right\}$ & $\left\{v_{2}\right\}$ \\
\hline$S P 3$ & $\left\{v_{5}, v_{7}\right\}$ & $\left\{v_{5}, v_{7}\right\}$ & $\left\{v_{6}\right\}$ & $\left\{v_{5}\right\}$ \\
\hline$S P 4$ & $\left\{v_{6}, v_{8}\right\}$ & $\left\{v_{6}\right\}$ & $\left\{v_{7}\right\}$ & $\left\{v_{7}\right\}$ \\
\hline$S P 5$ & $\left\{v_{9}\right\}$ & $\left\{v_{10}, v_{11}\right\}$ & $\left\{v_{8}\right\}$ & $\left\{v_{9}\right\}$ \\
\hline$S P 6$ & $\left\{v_{11}\right\}$ & & $\left\{v_{9}, v_{11}\right\}$ & $\left\{v_{10}\right\}$ \\
\hline$S P 7$ & & & $\left\{v_{10}\right\}$ & $\left\{v_{11}\right\}$ \\
\hline$S P$ S & \multicolumn{3}{|c|}{$v_{12}$} & \\
\hline
\end{tabular}

TABLE II. THE BEACONING SCHEDULE PRODUCED BY THE ALGORITHM SCBSD-PR FOR THE GRAPH GIVEN IN EXAMPLE 2. IN EACH ROW, "SP I" STANDS FOR THE I-TH SCHEDULING PERIOD.

shown in Fig. 4. That is, two nodes in $H$ are directly connected if and only if they are at most two-hop away in the communication topology.

The scheduling period $T=\{0,1,2,3\}$. The number inside a node represents the active time slot of the node. At time slot $0, U_{0}=\left\{v_{1}, v_{3}, v_{10}\right\}$ and $N\left(U_{0}\right)=$ $\left\{v_{2}, v_{4}, v_{5}, v_{6}, v_{7}, v_{8}, v_{9}, v_{11}\right\}$. We apply the algorithm SCBS$\operatorname{Pr}$ on the induced subgraph $H\left[N\left(U_{0}\right)\right]$ of $H$. The nodes in $N\left(U_{0}\right)$ is then partitioned into six independent sets $I_{01}=$ $\left\{v_{2}\right\}, I_{02}=\left\{v_{4}\right\}, I_{03}=\left\{v_{5}, v_{7}\right\}, I_{04}=\left\{v_{6}, v_{8}\right\}, I_{05}=$ $\left\{v_{9}\right\}$, and $I_{06}=\left\{v_{11}\right\}$. The nodes in $I_{01}, I_{02}, I_{03}, I_{04}, I_{05}$, and $I_{06}$ are scheduled to transmit at the time-slot 0 in the Scheduling Periods (SP) $1,2, \cdots, 6$, respectively. Similarly, at each time slot $i(1 \leq i \leq 3)$, we apply the strip-wise coloring algorithm SCBS-Pr on the induced subgraph $H\left[N\left(U_{i}\right)\right]$ of $H$. The beaconing schedule produced by the algorithm SCBSD-Pr is listed in the Table II. Each row lists the sets of nodes will be scheduled to transmit at the specified Scheduling Period (SP). Each column lists the sets of nodes that will be scheduled to transmit at the specified time-slot. The set of nodes in each entry is scheduled to transmit at the time-slot specified by the column at the scheduling period specified by the row. For example, the set of nodes $\left\{v_{6}, v_{8}\right\}$ is scheduled to transmit at time-slot 0 of the scheduling period 4 (SP 4). Totally, eight scheduling periods are required for all the twelve nodes to complete their beaconing operations based on the algorithm SCBSD-Pr.

The next theorem asserts the correctness of the above algorithm SCBSD-Pr and establishes an constant approximation bound for this algorithm.

Theorem 10: The algorithm SCBSD-Pr proposed above is correct and it uses at most $|T| \cdot(k(\rho)+1) \omega(H)$ colors. The performance ratio of this algorithm is at most $2(k(\rho)+1)$, which is at most 10 when $\rho=1$, and between 6 and 12 in general.

Proof: Every color is represented by a pair of positive integers $\left(\lambda_{1}, \lambda_{2}\right)$, where $\lambda_{1}$ represents a time slot in a scheduling period $T=\{0,1, \cdots,|T|-1\}$ and $\lambda_{2}$ represents one of the independent sets in the partition of the nodes in $N\left(U_{\lambda_{1}}\right)$ when applying the algorithm SCBS-Pr on the induced subgraph $H\left[N\left(U_{\lambda_{1}}\right)\right]$. Therefore, $0 \leq \lambda_{1} \leq|T|-1$ and $1 \leq \lambda_{2} \leq Y$. Thus, the algorithm SCBSD-Pr uses at most $Y|T|$ colors.

Next we prove the algorithm SCBSD-Pr is correct. Given any node $v \in V$. Assume that $v$ is active at the time slot $s$ for some $0 \leq s \leq|T|-1$. Then $v \in U_{s}$. When applying the algorithm SCBS-Pr on the induced subgraph $H\left[N\left(U_{s}\right)\right]$, the nodes in $N\left(U_{s}\right)$ is partitioned into $k_{s}$ independent sets $I_{s 1}, I_{s 2}, \cdots, I_{s k_{s}}$, for some $k_{s} \leq Y$ by Lemma 9. For each $1 \leq j \leq k_{s}$, the nodes in $I_{s j}$ transmit at the time slot $s$ in the $j$-th scheduling period. Since a sender can wake up at any time slot to transmit the message as long as the receiver node is awake and there is no collision for this transmission. Therefore, at the end of the $Y$-th scheduling period, the node $v$ received the messages from all of its neighbors. Thus, at the end of the $Y$-th scheduling period, the beaconing operations of all the networking nodes are complete.

Based on the lower bound obtained in Lemma 1, the performance ratio of the algorithm is at most

$$
\begin{aligned}
& \frac{|T| \cdot(k(\rho)+1) \omega(H)}{(\omega(H)-1)|T|} \\
& \leq(k(\rho)+1)(1+1 /(\omega(H)-1)) \\
& \leq 2(k(\rho)+1) .
\end{aligned}
$$

Therefore, by the upper and lower bounds for $k(\rho)$ obtained in Lemma 8, the algorithm achieves approximation bound at most 10 when $\rho=1$, and between 6 and 12 in general.

\section{Beaconing Schedule with Duty-Cycled Scenarios SubJect to Physical Interference}

We consider an instance of a multihop wireless network with duty-cycled scenarios under the SINR model specified by a finite planar set $V$ of nodes together with a uniform transmission power $P$ for each node $v \in V$. By proper 
scaling, we assume that the beaconing radius is one. Using the notations introduced in Section I for the SINR model, let

$$
R=\left(\frac{\eta P}{\sigma \xi}\right)^{1 / \kappa} \text {. }
$$

Then, a pair of nodes $u$ and $v$ can communicate with each other in the absence of interference if and only if $\|u v\| \leq R$. The value $R$ is thus referred to as the maximum transmission radius. Let $\lambda=1 / R$, i.e. $\lambda$ is the ratio of the beaconing radius and the maximum transmission radius. We assume $\lambda \leq 1-\varepsilon$ for some small positive constant $\varepsilon<1$. This assumption is valid in almost all practical applications of multihop wireless networks.

A set $I$ of nodes is said to be independent under the SINR model if (1) the mutual distances of the nodes in $I$ are greater than one, and (2) when all nodes in $I$ transmit simultaneously, the transmission by every node $u \in I$ can be received successfully by all nodes within the beaconing range of $u$. Then, any beaconing schedule is a partition of all the nodes in $V$ into independent sets.

Let $\zeta(x)$ denote the Riemann zeta function in the form $\zeta(x)=\sum_{j=1}^{\infty} \frac{1}{j^{x}}$, and let

$$
\rho=1+\left(\frac{\sigma(16 \zeta(\kappa-1)+8 \zeta(\kappa)-6)}{1-\lambda^{\kappa}}\right)^{1 / \kappa} .
$$

The following lemma, proved in [15], gives a sufficient condition for a set of nodes to be independent under the SINR model (see Lemma 10 in [15]).

Lemma 11: Assume $I$ is a set of nodes in $V$ whose mutual distances are greater than $\rho$. Then, $I$ is an independent set under the SINR model.

For any $r>0$, a set $I$ of nodes is an independent set of the $r$-disk graph on $V$ if and only if their mutual distances are greater than $r$. Therefore, by Lemma 11, any independent set of the $\rho$-disk graph on $V$ is an independent set under the SINR model. Let $G_{\rho}$ denote the $\rho$-disk graph on $V$. Note that any vertex coloring of $G_{\rho}$ is a partition of the nodes in $V$ into independent sets of $G_{\rho}$, each of which receives a distinct color. Thus, any vertex coloring of $G_{\rho}$ gives a beaconing schedule for the nodes in $V$ under the SINR model.

Since the smallest-degree-last ordering has the smallest inductivity, the beaconing schedule corresponding to the firstfit coloring of $G_{\rho}$ in the smallest-degree-last ordering is referred to as the first-fit beaconing schedule of the nodes in $V$ under the SINR model. The following lemma, proved in [15], gives the performance bound for the first-fit coloring of $G_{\rho}$ in the smallest-degree-last ordering (see Theorem 11 in [15]).

Lemma 12: The approximation bound of the first-fit coloring of $G_{\rho}$ in the smallest-degree-last ordering is at most $\mu(\rho)$.

Let opt be the minimum number of colors required in any vertex coloring of $G_{\rho}$. By Lemma 12, the first-fit coloring of $G_{\rho}$ in the smallest-degree-last ordering uses at most $\mu(\rho) \cdot$ opt colors.

Now we are ready to describe the algorithm to computer a First-Fit Beaconing Schedule with Duty-cycled scenarios under the Physical interference model (FFBSD-Ph). For any $u \in V$, let $N(u) \subseteq V \backslash\{u\}$ denote the set of all neighbors of $u$. For each time slot $0 \leq i \leq|T|-1$, let $U_{i}$ denote the set of all nodes in $V$ that are active in the time slot $i$ and

$$
N\left(U_{i}\right)=\underset{u \in U_{i}}{\cup} N(u) .
$$

At time slot 0 , we compute the first-fit coloring for the induced subgraph $G_{\rho}\left[N\left(U_{0}\right)\right]$ in the smallest-degree-last ordering. The nodes in $N\left(U_{0}\right)$ is then partitioned into independent sets

$$
I_{01}, I_{02}, \cdots, I_{0 k_{0}}
$$

of $G_{\rho}\left[N\left(U_{0}\right)\right]$ for some positive integer $k_{0}$. For each $1 \leq$ $j \leq k_{0}$, the nodes in $I_{0 j}$ transmit at the time slot 0 in the $j$-th scheduling period. In general, at each time slot $i(0 \leq$ $i \leq|T|-1$ ), we compute the first-fit coloring for the induced subgraph $G_{\rho}\left[N\left(U_{i}\right)\right]$ in the smallest-degree-last ordering. The nodes in $N\left(U_{i}\right)$ is then partitioned into independent sets

$$
I_{i 1}, I_{i 2}, \cdots, I_{i k_{i}}
$$

of $G_{\rho}\left[N\left(U_{i}\right)\right]$ for some positive integer $k_{i}$. For each $1 \leq j \leq$ $k_{i}$, the nodes in $I_{i j}$ transmit at the time slot $i$ in the $j$-th scheduling period.

The next theorem asserts the correctness of the above algorithm FFBSD-Ph and establishes an approximation bound for this algorithm.

Theorem 13: The algorithm FFBSD-Ph is correct. The performance ratio of the algorithm FFBSD-Ph under the SINR model is at most $\mu(\rho)|T|$.

Proof: Every color is represented by a pair of positive integers $\left(\lambda_{1}, \lambda_{2}\right)$, where $\lambda_{1}$ represents a time slot in a scheduling period $T=\{0,1, \cdots,|T|-1\}$ and $\lambda_{2}$ represents one of the independent sets in the partition of the nodes in $N\left(U_{\lambda_{1}}\right)$. These independent sets of $G_{\rho}\left[N\left(U_{\lambda_{1}}\right)\right]$ are obtained by computing 
the first-fit coloring for the induced subgraph $G_{\rho}\left[N\left(U_{\lambda_{1}}\right)\right]$ in the smallest-degree-last ordering. Therefore, $0 \leq \lambda_{1} \leq|T|-1$ and $1 \leq \lambda_{2} \leq \mu(\rho) \cdot$ opt. Thus, the algorithm FFBSD-Ph uses at most $|T| \cdot \mu(\rho) \cdot$ opt colors.

Next we prove the algorithm FFBSD-Ph is correct. Given any node $v \in V$. Assume that $v$ is active at the time slot $s$ for some $0 \leq s \leq|T|-1$. Then $v \in U_{s}$. When computing the first-fit coloring for the induced subgraph $G_{\rho}\left[N\left(U_{s}\right)\right]$ in the smallest-degree-last ordering, the nodes in $N\left(U_{s}\right)$ is partitioned into independent sets $I_{s 1}, I_{s 2}, \cdots, I_{s k_{s}}$ of $G_{\rho}\left[N\left(U_{s}\right)\right]$, where $k_{s} \leq \mu(\rho) \cdot o p t$. For each $1 \leq j \leq k_{s}$, the nodes in $I_{s j}$ transmit at the time slot $s$ in the $j$-th scheduling period. Since a sender can wake up at any time slot to transmit the message as long as the receiver node is awake and there is no collision for this transmission. Therefore, at the end of the $(\mu(\rho) \cdot o p t)$-th scheduling period, the node $v$ received the messages from all of its neighbors. Thus, at the end of the $(\mu(\rho) \cdot o p t)$-th scheduling period, the beaconing operations of all the networking nodes are complete.

\section{CONClusion ANd Future Work}

In this paper, we developed three efficient algorithms for MLBSDC. Under the protocol interference model, we first proposed a first-fit 74-approx. algorithm for MLBSDC with arbitrary interference radius. This algorithm achieves approximation bound at most 29 when all the nodes have uniform radius $\rho>1$, and at most 15 when $\rho=1$; then we developed another strip-coloring algorithm for MLBSDC that achieves approximation bound at most 10 when $\rho=1$, and between 6 and 12 in general. With the duty-cycled scenarios, the approximation bounds of all the previous known scheduling algorithms for any communication task (e.g., broadcast, data aggregation, etc.) depend on $|T|$, the length of a scheduling period. This is the first paper that develops constant-approx. algorithms for communication scheduling with the approximation bound independent of $|T|$ when the duty-cycled scenarios are taken into consideration. Finally, we developed an efficient approximation algorithm for MLBSDC under the physical interference model.

For future research directions, we can develop efficient constant-approx. algorithms for minimum-latency group communication scheduling (broadcast, data aggregation, etc.) with duty-cycled scenarios under the physical interference model.

Acknowledgement: This work was supported in part by the National Science Foundation of USA under grants HRD1238704, CNS-1219109, and CNS-1454770.

\section{REFERENCES}

[1] I. Chlamtac and A. Farago: Making Transmission Schedules Immune to Topology Changes in Multi-Hop Packet Radio Networks, IEEE/ACM Transactions on Networking, Vol. 2, No. 1, pp. 23-29, Feb. 1994.

[2] I. Chlamtac and S. Kutten: A spatial reuse tdma/fdma for mobile multihop radio nertworks, IEEE INFOCOM, pp. 389-394, March 1985.

[3] M. Chudnovsky, N. Robertson, P. Seymour and R. Thomas: The strong perfect graph theorem. Annals of Mathematics, 51-229. 2006.

[4] A. Ephremides and T.V. Truong: Scheduling Broadcasts in Multihop Radio Networks, IEEE Transactions on Communications, vol. 38, no. 4, pp. 456-60, April 1990.

[5] X. Jiao, W. Lou, J. Ma, J. Cao, X. Wang, X. Zhou: Minimum latency broadcast scheduling in duty-cycled multihop wireless networks. Parallel and Distributed Systems, IEEE Transactions on, 23(1), pp. 110117, 2012.

[6] J. Hong, J. Cao, W. Li, S. Lu, and D. Chen: Sleeping schedule-aware minimum latency broadcast in wireless ad hoc networks, in Proc. of IEEE ICC, 2009.

[7] K. Han, J. Luo, Y. Liu and A. Vasilakos: Algorithm Design for Data Communications in Duty-Cycled Wireless Sensor Networks: A Survey. Communications Magazine, IEEE, 51(7), 2013.

[8] S. C. H. Huang, P.-J. Wan, X. Jia, H. Du, and W. Shang: Minimumlatency broadcast scheduling in wireless ad hoc networks. The 26th IEEE International Conference on Computer Communications, pp. 733739. IEEE INFOCOM 2007.

[9] D. W. Matula and L. L. Beck: Smallest-last ordering and clustering and graph coloring algorithms, Journal of the Association of Computing Machinery, 30(3):417-427, 1983.

[10] S. Ramanathan and E.L. Lloyd: Scheduling algorithms for multi-hop radio networks, IEEE/ACM Transactions on Networking, vol. 1, pp. 166-172, April 1993.

[11] R. Nelson and L. Kleinrock, Spatial-TDMA: A collision-free multihop channel access protocol, IEEE Transactions on Communications, vol. 33, no. 9, pp. 934-944, Sep. 1985.

[12] A. Sen and M. L. Huson: A New Model for Scheduling Packet Radio Networks, ACM/Baltzer Journal Wireless Networks, 3 (1997), pp. 7182.

[13] A. Sen and E. Malesinska: Approximation Algorithms for Radio Network Scheduling, Proceedings of 35th Allerton Coneference on Communication, Control and Computing, Champaign, Illinois, pp. 573582, October, 1997.

[14] D.S. Stevens and M.H. Ammar: Evaluation of slot allocation strategies for TDMA protocols in packet radio networks, IEEE Military Сотmиnications Conference, pp. 835-839, 1990.

[15] P.-J. Wan, Z. Wang, H. Du, S. C.-H. Huang, and Z. Wan: FirstFit Scheduling for Beaconing in Multihop Wireless Networks, IEEE INFOCOM 2010.

[16] L. Wang, P.-J. Wan, and K. Young: Minimum-Latency Beaconing Schedule in Duty-Cycled Multihop Wireless Networks. This full version of the paper is available at http://www.cs.iit.edu/ /wan/Conference/dcfull.pdf

[17] P.-J. Wan, X. Xu, L. Wang, X. Jia, and E.K. Park: Minimum-Latency Beaconing Schedule in Multihop Wireless Networks, IEEE INFOCOM 2009.

[18] P.-J. Wan, C.-W. Yi, X. Jia, and D. Kim: Approximation Algorithms for Conflict-Free Channel Assignment in Wireless Ad Hoc Networks, Wiley Journal on Wireless Communications and Mobile Computing, 6(2):201211, March 2006.

[19] X. Xu, J. Cao, and P.-J. Wan: Fast Group Communication Scheduling in Duty-Cycled Multihop Wireless Sensor Networks, WASA 2012. 